\title{
Application of therapeutic plasma exchange in patients having severe fever with thrombocytope- nia syndrome
}

\author{
Jeong Rae Yoo, ${ }^{1,}$, Sun Hyung Kim, ${ }^{2,}$, Young Ree Kim², Keun Hwa Lee ${ }^{3}$, Won Sup Oh${ }^{4}$, and Sang Taek Heo
}

Departments of ${ }^{1}$ Internal Medicine, ${ }^{2}$ Laboratory Medicine, and ${ }^{3}$ Microbiology and Immunology, Jeju National University School of Medicine, Jeju; ${ }^{4}$ Department of Internal Medicine, Kangwon National University School of Medicine, Chuncheon, Korea

Received: June 23, 2016

Revised : December 18, 2016

Accepted: May 2, 2017

\section{Correspondence to}

Sang Taek Heo, M.D.

Division of Infectious Diseases,

Department of Internal Medi-

cine, Jeju National University

School of Medicine, 15 Aran 13-

gil, Jeju 63241, Korea

Tel: +82-64-754-8151

Fax: +82-64-717-1131

E-mail: neosangtaek@naver.com

*These authors contributed equally to this work.
Background/Aims: Severe fever with thrombocytopenia syndrome (SFTS) is a viral hemorrhagic fever with a high fatality rate. However, effective treatments for SFTS cases not responded to supportive therapy have not been established. Herein, we introduced the therapeutic plasma exchange (TPE) in SFTS patients in a tertiary hospital between 2013 and 2015.

Methods: TPE was performed in patients with rapidly progressing SFTS. Clinical, laboratory, and virological parameters were compared before and after TPE.

Results: Among 27 confirmed SFTS patients, two patients were treated with TPE and ribavirin combination in May 2013, then, 14 patients with rapidly progressing SFTS patients were treated with only TPE from June 2013 to September 2015: their median age was 58 years (interquartile range, 50 to 70 ) and eight (57.1\%) were male. Body temperature, pressure-adjusted heart rate, white blood cell and platelet counts, coagulation profile, serum creatinine, and multiple organ dysfunction score improved immediately after TPE. In addition, the mean cyclic threshold value of real-time reverse transcriptase polymerase chain reaction for SFTS virus after TPE (mean \pm standard deviation, $31.3 \pm 2.9$ ) was significantly higher than that before TPE $(26.5 \pm 2.9 ; p<0.001)$, indicating that serum viral loads decreased after TPE. Finally, 13 of 14 TPE-treated patients (92.8\%) recovered from rapidly progressing SFTS without sequelae.

Conclusions: SFTS patients treated with TPE showed improvements in clinical, laboratory, and virological parameters. These results suggest that TPE would be a therapeutic modality as rescue therapy in patients with rapidly progressing SFTS.

Keywords: Severe fever with thrombocytopenia syndrome; Plasma exchange; Therapeutics

\section{INTRODUCTION}

Severe fever with thrombocytopenia syndrome (SFTS) is an emerging hemorrhagic fever caused by a novel Phlebovirus in the Bunyaviridae family, named SFTS virus (SFTSV) [1]. Since 2009, more than 2,500 SFTS cases have been reported in China [2]. This febrile illness has also been reported outside China, such as Korea and Japan. SFTSV is usually transmitted to human through tick bites. However, human-to-human transmission has also been reported [3-5]. SFTSV was detected in human blood, tracheal and gastric aspirate, and urine. It can be transmitted by aerosol as a possible transmission route $[6,7]$. The wide distribution of SFTSV and the possibil- 
ity of human-to-human transmission make it a notable threat to public health worldwide.

The clinical spectrum of SFTS ranges from asymptomatic or self-limiting infection to life-threatening illness. The average case fatality rate of SFTS in China is $12 \%$ [8], but up to $30 \%$ in some areas [9]. The overall case fatality rate of SFTS is about $23.6 \%$ in South Korea [10]. Despite these high case fatality rates, no effective treatment for SFTS has been established.

In our previous report [11], therapeutic plasma exchange (TPE), combined with ribavirin, was successfully used to treat SFTS patients who had progressed rapidly to multiple organ dysfunction. In another study [12], ribavirin had no beneficial effect on the clinical outcome of SFTS patients. Then, we have applied TPE to SFTS patients presenting with rapid progression in Jeju National University Hospital since May 2013. Herein, we report on the use of TPE in SFTS patients in a tertiary hospital between 2013 and 2015.

\section{METHODS}

\section{Study subjects and definitions}

From May 2013 to July 2015, all consecutive patients over 18 years old who presented with fever (body temperature $\geq 38.0^{\circ} \mathrm{C}$ ) or history of fever and thrombocytopenia $(<150,000$ platelets $/ \mu \mathrm{L})$ were screened by taking a history focusing on fieldwork, outdoor activities, and tick bites. SFTS cases were defined as laboratory-confirmed SFTSV infection using a real-time reverse transcriptase polymerase chain reaction (RT-PCR) assay performed according to a standardized protocol in the Jeju Institute of Health and Environment.

Among all confirmed SFTS cases, TPE was applied to rapidly progressing patients, defined as severe thrombocytopenia $(<50,000$ platelets $/ \mu \mathrm{L})$, severe neutropenia (absolute neutrophil count $<500$ cells $/ \mu \mathrm{L}$ ), or clinical deterioration including altered mentality, disseminated intravascular coagulopathy, or septic shock despite conventional therapy. TPE was performed using the COBE Spectra Apheresis System (Terumo BCT, Lakewood, CO, USA), with acid citrate dextrose as an anticoagulant, and fresh frozen plasma as a plasma substitute. In each session, the volume of plasma substitute was calculated based on the height, weight, and hematocrit of the pa- tient. The study protocol was approved by the Institutional Review Board of Jeju National University Hospital (JNUH 1310010) and informed consent was obtained from all participants.

\section{Data collection}

We collected information about epidemiologic data, clinical features, laboratory results, and treatment outcomes. The multiple organ dysfunction (MOD) score, which was originally proposed by Marshall et al. [13] was serially calculated for the assessment of organ dysfunction based on six criterias. In addition, to evaluate the efficacy of TPE, clinical, laboratory, and virological parameters, including the cyclic threshold (Ct) value of real-time RT-PCR for SFTSV as a surrogate measure of serum viral load, within 48 hours before TPE, were compared with those within 48 hours after TPE. Real-time RT-PCR of partial S segments was performed respectively for molecular diagnosis of SFTSV [14,15].

\section{Statistical analysis}

Results of categorical variables are presented as frequencies or proportions, and those of continuous variables as means and standard deviation (SD) or medians and interquartile range (IQR). To compare clinical, laboratory, and virological parameters within 48 hours before and after TPE, a paired $t$ test was used for parametric data, and Wilcoxon signed-rank test for nonparametric data. In addition, Mann-Whitney $U$ test was used for nonparametric data between the TPE and non-TPE groups. SPSS version 20.0 (IBM Co., Armonk, NY, USA) was used for the statistical analyses. A two-tailed $p<0.05$ was considered to indicate statistical significance.

\section{RESULTS}

From May 2013 to July 2015, real-time RT-PCR for SFTSV was performed on samples from 41 adult patients presenting to our hospital with either fever or history of fever and thrombocytopenia. Of these, 27 patients were confirmed as having SFTSV infection: their median age was 60 years (IQR, 50 to 67 ) and 14 (53.8\%) were male. Of the 27 patients, the first two patients died of multiple organ dysfunction and hemorrhage with fully supportive care in intensive care unit in May 2013. The next, two 
patients recovered with TPE and ribavirin from rapidly progressing SFTS in May 2013. The others 23 patients were diagnosed with SFTS after the use of only TPE was implementated from June 2013 to September 2015. Fourteen $(51.6 \%)$ were treated with only TPE due to severe thrombocytopenia and severe neutropenia $(n=6)$, severe thrombocytopenia $(n=3)$, altered mentality $(n=2)$, severe neutropenia $(\mathrm{n}=2)$, and severe thrombocytopenia and altered mentality $(n=1)$.

\section{Clinical characteristics of TPE group}

The characteristics of the 14 TPE-treated and nine nonTPE patients are summarized in Table 1. The median age of the 14 TPE-treated patients was 58 years (IQR, 50 to 70 ) and eight (57.1\%) were male. All patients had a recent history of fieldwork or outdoor activities, and about $80 \%$ were previously healthy. The median interval from symptom onset to initial presentation was 4 days (IQR, 3 to 10$)$. At the time of initial presentation, more than $70 \%$ of these patients had gastrointestinal symptomsincluding anorexia, nausea, vomiting, and diarrhea, and more than $20 \%$ showed altered mentality. Leukopenia was the most common laboratory finding, followed in order by increased levels of tissue damage indicator enzymes, such as lactate dehydrogenase, alanine aminotransferase, or creatine phosphokinase. Of the 14 patients, only two (14.3\%) had MOD syndrome at the time of initial presentation. The interval from symptom onset to initiation of TPE was median 6 days (IQR, 4.8 to 8.3). All patients were admitted to the intensive care unit for implementation of TPE. One patient (7.1\%) was mechanically ventilated.

\section{Comparison of clinical characteristics and virological parameters before and after TPE}

The median volume of plasma removed during TPE was 7,154 mL (IQR, 5,261 to 8,015), and the median number of TPE sessions was three (Supplementary Table 1). After completion of TPE, all patients had defervescence within 3 days and 13 (92.8\%) showed complete recovery of platelet and white blood cell (WBC) counts within 7 days. Thirteen of the 14 TPE-treated patients (92.9\%) recovered completely without sequelae, the other $(n=1)$ died of bleeding or multiple organ failure. No adverse events associated with TPE were reported. Clinical, laboratory, and virological parameters within 48 hours before
TPE were compared with those within 48 hours after TPE (Table 2). The median MOD score decreased from 4 (IQR, 3 to 4) to 2 (IQR, 1 to 2) just before initiation of TPE. However, 13 patients showed decreased MOD score after TPE. Of the six criteria comprising the MOD score, the mean pressure-adjusted heart rate (PAR) after TPE was significantly lower than that before TPE $(9.6 \pm 4.3 \mathrm{vs.} 8.0$ $\pm 3.2, p=0.014)$ and mean serum creatinine after TPE (1.0 $\pm 0.2 \mathrm{mg} / \mathrm{dL}$ vs. $0.9 \pm 0.2 \mathrm{mg} / \mathrm{dL}, p=0.011)$. The median platelet count increased from 37,000 cells/ $\mu \mathrm{L}$ before TPE to 76,000 cells $/ \mu \mathrm{L}$ after TPE. Median WBC and neutrophil counts and coagulation profile improved after T'PE. The mean Ct value of real-time RT-PCR for SFTSV after TPE was significantly higher than that before TPE (31.3 \pm 2.9 vs. $26.5 \pm 2.9, p<0.001)$, indicating that serum viral loads decreased after TPE. One patient had an increase in MOD score, change of viral load was not checked. Confirmation interval and laboratory results before and after TPE in rapidly progressive SFTS are presented in the Supplementary Table 1.

\section{Comparison of clinical characteristics between TPE and non-TPE groups}

During the implementation period where TPE was used, there were nine patients not treated using TPE group. Their median age was 59 years (IQR, 49.5 to 68.6) and five $(55.6 \%)$ were male in the non-TPE group. The median interval from symptom onset to initial presentation showed a longer trend in the TPE group than the non-TPE group. Neutropenia was significantly worse in the TPE group. Thrombocytopenia and coagulopathy showed a progressive trend in the TPE group. Other laboratory findings were not significant in the initial presentation between both groups. Median time to confirm SFTS was significantly shorter in the TPE group than in the non-TPE group (1 day vs. 2 days, $p<0.001$ ).

\section{DISCUSSION}

The Korea Centers for Disease Control and Prevention reported that 144 SFTS patients were confirmed, and 44 of these died in Korea (23.6\%) from 2012 to 2015 [10]. The highest prevalence was on Jeju Island, which is located about $80 \mathrm{~km}$ southwest of the Korean mainland. There 27 patients were diagnosed as SFTSV infection, with a 
Table 1. Comparison of clinical characteristics of SFTS patients between TPE group and non-TPE group

\begin{tabular}{|c|c|c|c|}
\hline Characteristic & TPE $(n=14)$ & Non-TPE $(n=9)$ & $p$ value \\
\hline \multicolumn{4}{|l|}{ Epidemiology } \\
\hline Male sex & $8(57.1)$ & $5(55.6)$ & 0.94 \\
\hline Age, yr & $58.0(50.0-69.5)$ & $58.5(49.5-68.6)$ & 0.82 \\
\hline Recent history of fieldwork or outdoor activities & $14(100.0)$ & $9(100.0)$ & 1.00 \\
\hline Recent history of tick bite & $8(57.1)$ & $5(55.6)$ & 0.94 \\
\hline Presence of one or more comorbid conditions & $5(35 \cdot 7)$ & $4(44 \cdot 4)$ & 0.68 \\
\hline \multicolumn{4}{|l|}{ Clinical features at the time of initial presentation } \\
\hline Interval from symptom onset to initial presentation, day & $4(3-10)$ & $3(2-4)$ & 0.08 \\
\hline Fever & $14(100.0)$ & $8(88.9)$ & 0.21 \\
\hline Headache & $7(50.0)$ & $7(77 \cdot 7)$ & 0.21 \\
\hline Myalgia & $9(64.3)$ & $7(77.7)$ & 0.74 \\
\hline Anorexia, nausea, vomiting & $11(78.6)$ & $6(66.7)$ & 0.65 \\
\hline Diarrhea & $7(50.0)$ & $2(22.2)$ & 0.50 \\
\hline Altered mentality & $3(21.4)$ & o & 0.15 \\
\hline Hypotension (systolic blood pressure < $90 \mathrm{mmHg}$ ) & $2(14.3)$ & o & 0.83 \\
\hline Tachycardia (heart rate $>90$ beats/min) & $5(35 \cdot 7)$ & $3(33 \cdot 3)$ & 0.93 \\
\hline Tachypnea (respiratory rate > 20 breaths/min) & $3(21.4)$ & $3(33 \cdot 3)$ & 0.83 \\
\hline Lymphadenopathy & $2(14 \cdot 3)$ & $3(33 \cdot 3)$ & 0.04 \\
\hline \multicolumn{4}{|l|}{ Laboratory findings at the time of initial presentation } \\
\hline Leukopenia (WBC counts < 4,0oo cells/ $\mu \mathrm{L}$ ) & $13(92.9)$ & $6(75.0)$ & 0.25 \\
\hline Neutropenia (neutrophil counts $<1,500$ cells $/ \mu \mathrm{L}$ ) & $9(64 \cdot 3)$ & $1(11.1)$ & 0.01 \\
\hline Thrombocytopenia (platelet counts $<100,000$ cells $/ \mu \mathrm{L}$ ) & $10(71.4)$ & $3(33.3)$ & 0.08 \\
\hline Coagulopathy (PT INR $>1.3$ or aPTT $>40 \mathrm{sec}$ ) & $10(71.4)$ & $2(28.6)$ & 0.07 \\
\hline Azotemia $(\mathrm{BUN}>20 \mathrm{mg} / \mathrm{dL}$ or creatinine $>1.5 \mathrm{mg} / \mathrm{dL})$ & $3(21.4)$ & $1(11.1)$ & 0.53 \\
\hline Increased level of aspartate aminotransferase ( $>50 \mathrm{U} / \mathrm{L}$ ) & $12(85.7)$ & $6(75.0)$ & 0.54 \\
\hline Increased level of lactate dehydrogenase (> 350 U/L) & $12(85.7)$ & $8(100.0)$ & 0.27 \\
\hline Increased level of creatine kinase (> 200 U/L) & $10(71.4)$ & $4(50.0)$ & 0.33 \\
\hline MOD score at the time of initial presentation & $2(1-3)$ & $N A^{a}$ & \\
\hline Use of vasopressors & $1(7.1)$ & o & 0.42 \\
\hline Use of antibiotic agents & $14(100.0)$ & $14(100.0)$ & 1.00 \\
\hline Admission to ICU & $14(100.0)$ & o & $<0.01$ \\
\hline Length of ICU stay, day & $5(3-6)$ & NA & \\
\hline Mechanical ventilation & $1(7.1)$ & o & 0.42 \\
\hline Continuous renal replacement therapy & $1(7.1)$ & o & 0.42 \\
\hline Length of hospital stay, day & $9.5(8-12)$ & $7(5-12)$ & 0.61 \\
\hline In-hospital fatality & $1(7.1)$ & o & 0.42 \\
\hline
\end{tabular}

Values are presented as number (\%) or median (interquartile range).

SFTS, severe fever with thrombocytopenia syndrome; TPE, therapeutic plasma exchange; WBC, white blood cell; PT, prothrombin time; INR, international normalized ratio; aPTT, activated partial thromboplastin time; BUN, blood urea nitrogen; $\mathrm{U} / \mathrm{L}$, units/liter; MOD, multiple organ dysfunction; NA, not applicable; ICU, intensive care unit.

${ }^{a}$ Non-TPE group was not calculated a MOD score, because they were not applied a central venous catheter and other parameters. 
Table 2. Comparison of clinical, laboratory, and virological parameters in 14 SFTS patients within 48 hours before and after therapeutic plasma exchange

\begin{tabular}{|c|c|c|c|}
\hline Parameter & Within 48 hours before TPE & Within 48 hours after TPE & $p$ value \\
\hline Temperature, ${ }^{\circ} \mathrm{C}$ & $37.7 \pm 0.8$ & $36.9 \pm 0.7$ & $<0.001^{\mathrm{a}}$ \\
\hline PAR & $9 \cdot 6 \pm 4 \cdot 3$ & $8.0 \pm 3.2$ & $0.014^{\mathrm{a}}$ \\
\hline $\mathrm{PaO}_{2} / \mathrm{FiO}_{2}$ & $457.1(386.9-625.7)$ & $433.3(349.8-483.3)$ & 0.116 \\
\hline Glasgow coma scale & $15(14-15)$ & $15(14-15)$ & 0.786 \\
\hline WBC, cells $/ \mu \mathrm{L}$ & $1,000(800-1,820)$ & $3,650(2,355-7,050)$ & $<0.001$ \\
\hline Neutrophil, cells $/ \mu \mathrm{L}$ & $375(258-685)$ & $1,060(501-4,618)$ & 0.001 \\
\hline Platelet, platelets $/ \mu \mathrm{L}$ & $37,000(29,000-48,000)$ & $76,000(63,000-108,000)$ & $<0.001$ \\
\hline PT, INR & $1.08 \pm 0.15$ & $1.01 \pm 0.13$ & $0.006^{\mathrm{a}}$ \\
\hline aPTT, sec & $46.5(39.5-56.3)$ & $32.5(30.5-37.3)$ & 0.049 \\
\hline Creatinine, mg/dL & $1.0 \pm 0.2$ & $0.9 \pm 0.2$ & $0.011^{a}$ \\
\hline Total bilirubin, mg/dL & $0.4(0.3-0.6)$ & $0.4(0.4-0.6)$ & 0.242 \\
\hline Aspartate aminotransferase, U/L & $234(134-271)$ & $258(81-315)$ & 0.682 \\
\hline Lactate dehydrogenase, U/L & $1,060(1,026-1,354)$ & $940(718-1,382)$ & 0.234 \\
\hline Creatine kinase, $\mathrm{U} / \mathrm{L}$ & $876(339-1,207)$ & $287(128-985)$ & 0.044 \\
\hline MOD score & $4(3-4)$ & $2(1-2)$ & 0.007 \\
\hline Ct of RT-PCR for SFTSV & $26.5 \pm 2.9$ & $31.3 \pm 2.9$ & $<0.001^{\mathrm{a}}$ \\
\hline
\end{tabular}

Values are presented as mean $\pm \mathrm{SD}$ or median (interquartile range).

SFTS, severe fever with thrombocytopenia syndrome; TPE, therapeutic plasma exchange; PAR, pressure-adjusted heart rate; $\mathrm{PaO}_{2}$, partial pressure of arterial oxygen; $\mathrm{FiO}_{2}$, fraction of inspired oxygen; WBC, white blood cell; PT, prothrombin time; INR, international normalized ratio; aPTT, activated partial thromboplastin time; U/L, units/liter; MOD, multiple organ dysfunction; Ct, cyclic threshold value; RT-PCR, reverse transcription polymerase chain reaction; SFTSV, severe fever with thrombocytopenia syndrome virus.

${ }^{\text {a }}$ Paired $t$ test was used to compare two means of parametric data.

case fatality rate of $14.8 \%$, up to the 2015 implementation of TPE. Excluding Jeju Island cases, the case-fatality rate of SFTS patients on the Korean mainland was up to 40.0\%. After TPE implementation in our hospital, the case fatality rate was $1 / 23$ (4.3\%). The patient died with multiple organ failure within 7 days after TPE. Following TPE implementation, the case fatality rate of SFTS patients on Jeju Island was lower than that on the Korean mainland as geographical controls (4.3\% vs. $28 \%$ ). However, we do not know whether application of plasma exchange actually influenced the improvement, or self remission due to fully supportive management through early diagnosis. So, our data has a little evidence exists to support its use. Other reported experimental therapies in SFTS include convalescent plasma therapy [16], the inhibitory effects in vitro [17], and the combination of treatment intravenous immunoglobulin and corticosteroid [18]. However, the efficacy of these approaches- remains controversial, and conservative treatment is effective for mild cases.

Currently, there is a need for an effective therapeutic modality to treat cases that will otherwise be fatal. Even if the pathogenesis of SFTS is not fully understand, cytokine-mediated inflammatory response plays an important role in progressive SFTS [19]. TPE may remove toxic compounds, such as cytokines, and toxic mediators in patients with SFTS. This suggests that TPE may modulate the cytokine storm in fatal SFTS patients. TPE is an extracorporeal blood purification technique designed to remove high-molecular-weight substances from plasma [20]. It has been reported to be of benefit in patients infected with several viruses. In three children having severe influenza complicated by respiratory failure and hemodynamic instability, clinical and laboratory improvements and a favorable outcome were achieved using TPE [21]. In addition, TPE was used in pa- 
tients with Crimean-Congo hemorrhagic fever (CCHF) caused by a Nairovirus of the same Bunyaviridae family as SFTSV $[22,23]$. After implementation of TPE, these patients showed significant improvements in clinical and laboratory parameters and recovered completely from CCHF. In this study, most SFTS patients also exhibited rapid improvements in clinical and laboratory parameters after TPE, including body temperature, PAR, WBC, neutrophil and platelet counts, coagulation profile, serum creatinine, and MOD score. Moreover, most of these improvements occurred within 48 hours after implementation of TPE. In particular, all TPE-treated patients showed defervescence within 3 days after initiation of TPE. Therapeutic modalities to reduce viral loads are potential treatments for patients with severe viral infection. Ribavirin showed antiviral activity in vitro [17], but it had no beneficial effect on viral loads, platelet counts, or clinical outcomes of SFTS patients [12]. Indeed, adverse events associated with ribavirin have been reported. In this study, most SFTS patients showed a significant decrease in serum viral loads as well as rapid improvements in clinical and laboratory parameters after implementation of TPE. The main cause of death in SFTS patients has been reported to be bleeding or multiple organ failure [8]. We speculate that TPE may help alleviate the deterioration of patients with rapidly progressing SFTS. However, cautious interpretation of TPE implementation is necessary because these therapies may have nothing to do with patient recovery. Although we did not observe any adverse effects of TPE, TPE can deplete coagulation factors or immunoglobulins, produced hypocalcemia and metabolic alkalosis, and cause complications related to vascular catheterization [24,25].

This study had several limitations. First, the noncomparative design resulted in limitations in concluding that TPE can reduce case-fatality rates in patients with rapidly progressing SFTS. Although clinical, laboratory and virological recovery were evident in the TPE group, we do not know whether the TPE actually had a role in the improved outcome. However, TPE can be used as a potential rescue therapy for severe SFTS patients, given the observation of improved survival rates in patients with rapidly progressing SFTS and the continuing controversy over other experimental therapies. Second, a previous study regarding the dynamic tracking of disease progress identified three distinct stages of SFTS-fever, MOD, and convalescence [3,26]. The MOD stage is characterized by a progressive exacerbation of MOD in fatal cases or a self-limiting process in survivors. Although TPE should be implemented in patients who are likely to die of SFTS, identifying those at high risk of death among all SFTS patients is difficult, even at the MOD stage. Because, a clinical course is mild in the non-TPE group, we did not calculate a MODS due to the invasive monitoring was unnecessary. Instead, patients with rapidly progressing SFTS, defined as having severe thrombocytopenia, severe neutropenia, or clinical deterioration despite conventional therapy after initial presentation, were presently identified as those in whom TPE should be implemented. However, we cannot rule out completely the possibility of inclusion of mild or self-limiting cases. Finally, although this study was conducted in a prospective manner, some variables regarding MOD score were retrospectively obtained by reviewing medical records and the Ct values of real-time RT-PCR for SFTSV were measured at the same time obtaining paired serum samples from each patient. Therefore, the decision to implement TPE could not be determined in accordance with the results of these parameters.

In conclusion, clinical, laboratory, and virological parameters of patients with rapidly progressing SFTS improved after implementation of TPE. In addition, considering the relatively low case-fatality rate of TPE-treated patients based on epidemiological data, TPE may lead to a favorable outcome in patients with rapidly progressing SFTS by improving these parameters. However, comparative studies of large sample size to confirm the effects of T'PE on the clinical outcomes of SFTS patients are warranted.

\section{KEY MESSAGE}

1. Severe fever with thrombocytopenia syndrome (SFTS) is a new emerging viral hemorrhagic fever disease.

2. There is no appropriate treatment for SFTS patients until now.

3. Therapeutic plasma exchange (TPE) showed improving results in rapid progressive SFTS patients.

4. TPE might be a therapeutic method in rapidly progressing SFTS patients. 


\section{Conflict of interest}

No potential conflict of interest relevant to this article was reported.

\section{Acknowledgments}

This work was done while the author's his research year of Jeju National University in 2015.

\section{REFERENCES}

1. Yu XJ, Liang MF, Zhang SY, et al. Fever with thrombocytopenia associated with a novel bunyavirus in China. N Engl J Med 2011;364:1523-1532.

2. Liu Q, He B, Huang SY, Wei F, Zhu XQ. Severe fever with thrombocytopenia syndrome, an emerging tick-borne zoonosis. Lancet Infect Dis 2014;14:763-772.

3. Gai ZT, Zhang Y, Liang MF, et al. Clinical progress and risk factors for death in severe fever with thrombocytopenia syndrome patients. J Infect Dis 2012;206:1095-1102.

4. Tang X, Wu W, Wang H, et al. Human-to-human transmission of severe fever with thrombocytopenia syndrome bunyavirus through contact with infectious blood. J Infect Dis 2013;207:736-739.

5. Kim WY, Choi W, Park SW, et al. Nosocomial transmission of severe fever with thrombocytopenia syndrome in Korea. Clin Infect Dis 2015;60:1681-1683.

6. Gong Z, Gu S, Zhang Y, et al. Probable aerosol transmission of severe fever with thrombocytopenia syndrome virus in southeastern China. Clin Microbiol Infect 2015;21:1115-1120.

7. Jeong EJ, Song JY, Lim CS, et al. Viral shedding from diverse body fluids in a patient with severe fever with thrombocytopenia syndrome. J Clin Virol 2016;80:33-35.

8. Liu S, Chai C, Wang C, et al. Systematic review of severe fever with thrombocytopenia syndrome: virology, epidemiology, and clinical characteristics. Rev Med Virol 2014;24:90-102.

9. Deng B, Zhou B, Zhang S, et al. Clinical features and factors associated with severity and fatality among patients with severe fever with thrombocytopenia syndrome Bunyavirus infection in Northeast China. PLoS One 2013;8:e80802.

10. Korea Centers for Disease Control and Prevention. Severe fever with thrombocytopenia syndrome (SFTS), and statistical system of notifiable disease surveillance system
(2013-2015) [Internet]. Cheongju (KR): Korea Centers for Disease Control and Prevention, 2017 [cited 2017 Sep 15]. Available from: https://is.cdc.go.kr.

11. Oh WS, Heo ST, Kim SH, Choi WJ, Han MG, Kim JY. Plasma exchange and ribavirin for rapidly progressive severe fever with thrombocytopenia syndrome. Int J Infect Dis 2014;18:84-86.

12. Liu W, Lu QB, Cui N, et al. Case-fatality ratio and effectiveness of ribavirin therapy among hospitalized patients in china who had severe fever with thrombocytopenia syndrome. Clin Infect Dis 2013;57:1292-1299.

13. Marshall JC, Cook DJ, Christou NV, Bernard GR, Sprung CL, Sibbald WJ. Multiple organ dysfunction score: a reliable descriptor of a complex clinical outcome. Crit Care Med 1995;23:1638-1652.

14. Heid CA, Stevens J, Livak KJ, Williams PM. Real time quantitative PCR. Genome Res 1996;6:986-994.

15. Zhang YZ, He YW, Dai YA, et al. Hemorrhagic fever caused by a novel Bunyavirus in China: pathogenesis and correlates of fatal outcome. Clin Infect Dis 2012;54:527-533.

16. Park SY, Choi W, Chong YP, et al. Use of plasma therapy for severe fever with thrombocytopenia syndrome encephalopathy. Emerg Infect Dis 2016;22:1306-1308.

17. Shimojima M, Fukushi S, Tani H, et al. Effects of ribavirin on severe fever with thrombocytopenia syndrome virus in vitro. Jpn J Infect Dis 2014;67:423-427.

18. Kim UJ, Kim DM, Ahn JH, et al. Successful treatment of rapidly progressing severe fever with thrombocytopenia syndrome with neurological complications using intravenous immunoglobulin and corticosteroid. Antivir Ther 2016;21:637-640.

19. Deng B, Zhang S, Geng Y, et al. Cytokine and chemokine levels in patients with severe fever with thrombocytopenia syndrome virus. PLoS One 2012;7:e41365.

20. Schwartz J, Winters JL, Padmanabhan A, et al. Guidelines on the use of therapeutic apheresis in clinical practiceevidence-based approach from the Writing Committee of the American Society for Apheresis: the sixth special issue. J Clin Apher 2013;28:145-284.

21. Patel P, Nandwani V, Vanchiere J, Conrad SA, Scott LK. Use of therapeutic plasma exchange as a rescue therapy in $2009 \mathrm{pH} 1 \mathrm{~N} 1$ influenza A: an associated respiratory failure and hemodynamic shock. Pediatr Crit Care Med 2011;12:e87-e89.

22. Kurnaz F, Metan G, Coskun R, Kaynar L, Eser B, Doganay M. A case of Crimean-Congo haemorrhagic fever suc- 
cessfully treated with therapeutic plasma exchange and ribavirin. Trop Doct 2011;41:181-182.

23. Meco BC, Memikoglu O, Ilhan O, et al. Double filtration plasmapheresis for a case of Crimean-Congo hemorrhagic fever. Transfus Apher Sci 2013;48:331-334.

24. Pearl RG, Rosenthal MH. Metabolic alkalosis due to plasmapheresis. Am J Med 1985;79:391-393.
25. Mokrzycki MH, Kaplan AA. Therapeutic plasma exchange: complications and management. Am J Kidney Dis 1994;23:817-827.

26. Cui N, Bao XL, Yang ZD, et al. Clinical progression and predictors of death in patients with severe fever with thrombocytopenia syndrome in China. J Clin Virol 2014;59:12-17. 


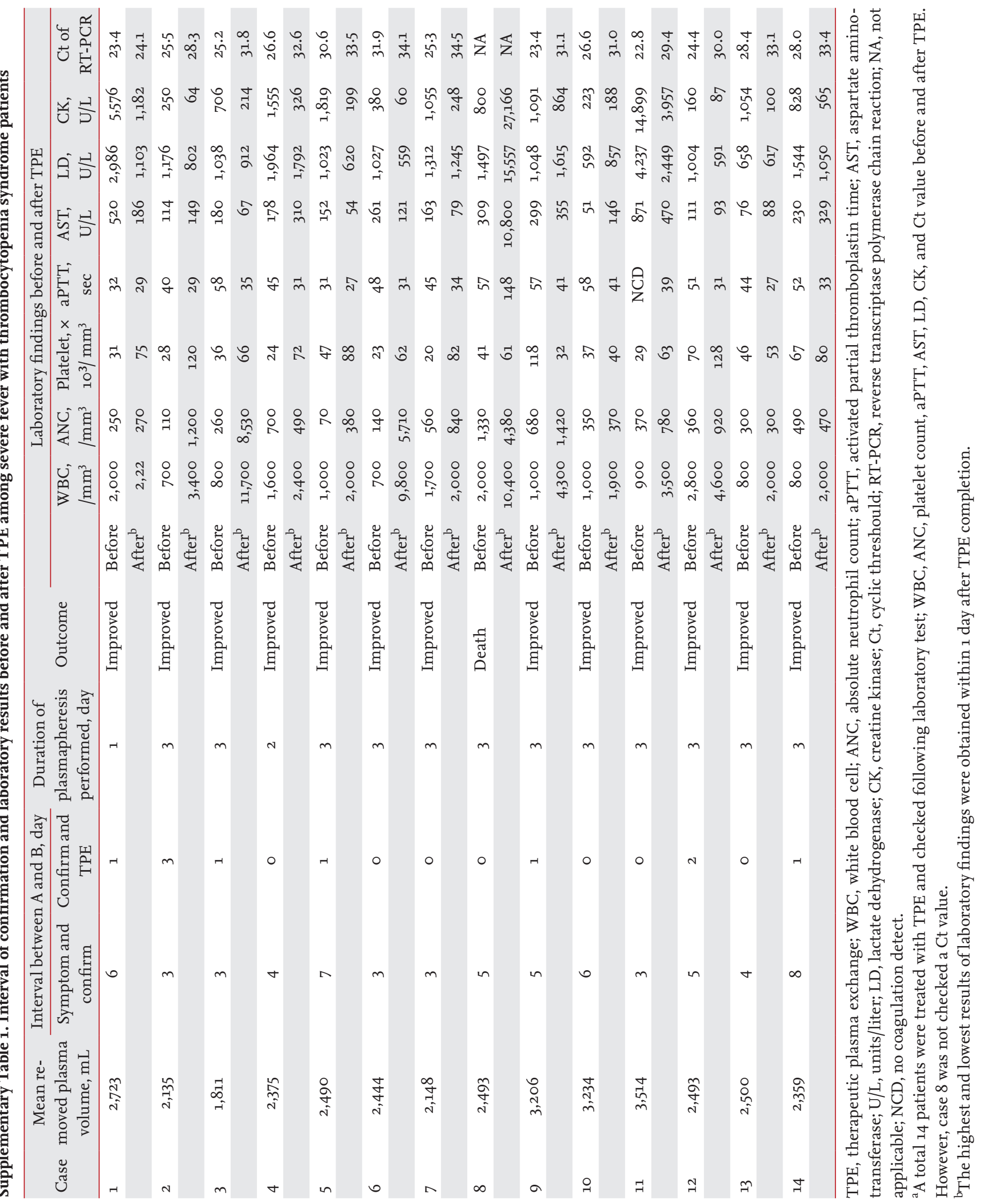

